\title{
Accessing Mechanical Properties of Superelastic Materials from Microindentation Data by Computer Modelling
}

\author{
Yuriy Perlovich, Margarita Isaenkova, Dmitry Zhuk ${ }^{+}$and Olga Krymskaya \\ Center of Nuclear Systems and Materials, National Research Nuclear University MEPhI, Moscow, Russia \\ 115409
}

\begin{abstract}
Indentation of superelastic alloys with stress-induced phase transformation analysed in this paper with use of finite element modelling method. Several series of indentation simulations performed with spherical indenters of various geometries for different material's mechanical properties data sets. Proposed a function for determination of mechanical properties of superelstic materials.
\end{abstract}

Keywords: nanoindentation, superelastic alloys, stress-induced phase transformation, spherical indenter, finite element method.

\section{Introduction}

Superelastic (SE) is a common name for materials which can deform recoverably to high strain rates. Despite of its name, the deformation of such materials is mostly nonelastic but conducted with phase transformation. As a subdivision of shape memory (SM) materials, SE materials share same physical processes what lead to their unique properties. Presence of phase transformation from martensite to austenite, depending on temperature and stress state, can yield material SE effect, one or two way shape memory effect. With different temperatures the material with one chemical composition and processing can possess SE or shape memory properties. Also, material can change phase or shape depending on temperature which is known as two way shape memory effect.

Existence of high temperature martensite phase is known in metallurgy for more than 100 years, yet, recovery of deformation under heating (SM effect) and high strain deformation with phase transition (SE effect) was discovered only in 1932 [1]. First discovered alloy which has such properties was Ni-Ti with close to equal composition of both atoms. Although with even small change in composition (1-2\%) the material properties (phase change stresses and transition temperatures) can change significantly. Until this time, NiTi is still the most used alloy in SM material group. It is used in aerospace, nanotechnology, medicine and in many more fields [2] but more or less it is replaced with cheaper or better materials. Ti-Nb$\mathrm{Zr}$ have higher biocompatibility so it is getting more widely used in medicine [3]. SM composites have much lower elastic modulus and there are also many applications for its properties.

Testing process of pressing spherical indenter to SE material is researched in this paper. Indentation was popular in 20th century and still popular now for testing of material's hardness which is rather complex measurement depending on elastic and plastic characteristics, ductility, viscosity and many more. Indentation technologies greatly improved over last several decades and some work have been done in dividing one characteristic from another [4]. For SE materials it is much more difficult to output mechanical characteristics from only load-displacement (P-h) diagram due to much more complex nature, compared to plastic materials. Schematic example of uniaxial extension/compression diagram of SE material obtained

\footnotetext{
${ }^{+}$Corresponding author. Tel.: +7(915) 084-6940

E-mail address:_dimazhuk@gmail.com.
} 
from experiment is shown on figure 1 to the left. The one used for finite element model (FEM) is also shown on the figure 1 to the right.
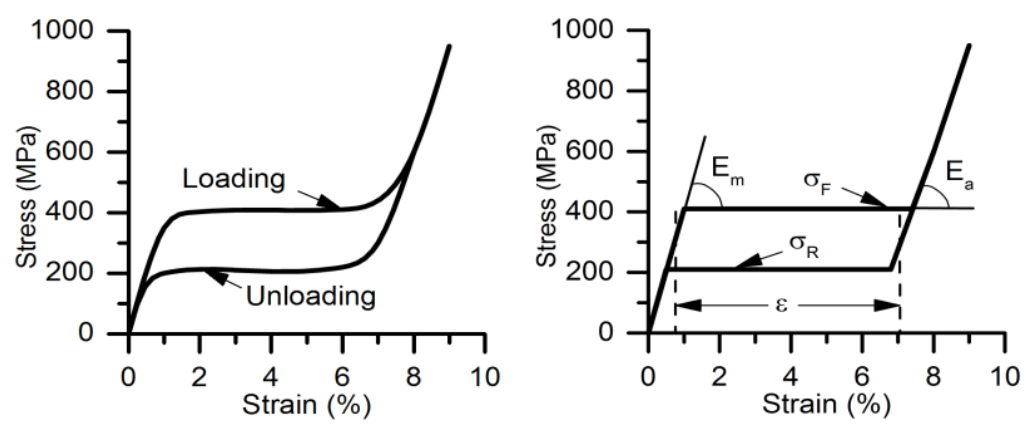

Fig. 1: Typical schematic uniaxial extension/compression diagram of SE material (left) and method of its approximation used in this work (right).

\section{Finite Element Modelling}

As shown on figure 1, for simplification, in constitutive model of SE material several assumptions were made:

(1) Stresses of phase transformations start and finish does not differ much, which is true for many materials and this difference does not affect result significantly.

(2) Plastic or unrecoverable strains are small. This can be arranged by varying the load or spherical indenter radius so the load is high enough for chosen indenter to evoke phase transformation of a significant volume. At the same time, it should not be too high to lead to unrecoverable plastic deformations.

(3) Obtained material properties correspond to material compression because indentation is mostly a compression load. For comparing results from indentation with uniaxial extension test, material should perform similarly in extension and compression.

(4) Material does not have sharp texture or significant anisotropy.

Constitutive model developed by Auricchio research group implemented in commercially available software was used in this work [5]. Although the assumptions above are not required for use of this model, they needed to limit number of variables for relations between material properties and indentation results. This will make these relations easier and more stable to input. Several material properties of FE model were varied for understanding of impact on indentation results. It includes forward transformation stress $\left(\sigma_{\mathrm{F}}\right)$, reverse transformation stress $\left(\sigma_{\mathrm{R}}\right)$, maximum transformation strain $(\varepsilon)$. These are key variables characterising a SE properties of material.

Constructed model included 2526 eight-node two-dimensional elements for axisymmetric modelling of SE modelling of microindentation (Fig. 2). It is possible to use two-dimensional elements without loss of accuracy because load and distribution of stresses in material are axisymmetric. This makes calculation faster by many times. In calculations transient solver was used, which means that each substep with increasing and decreasing after reaching the maximum load was calculated in chronological order for each material property set.
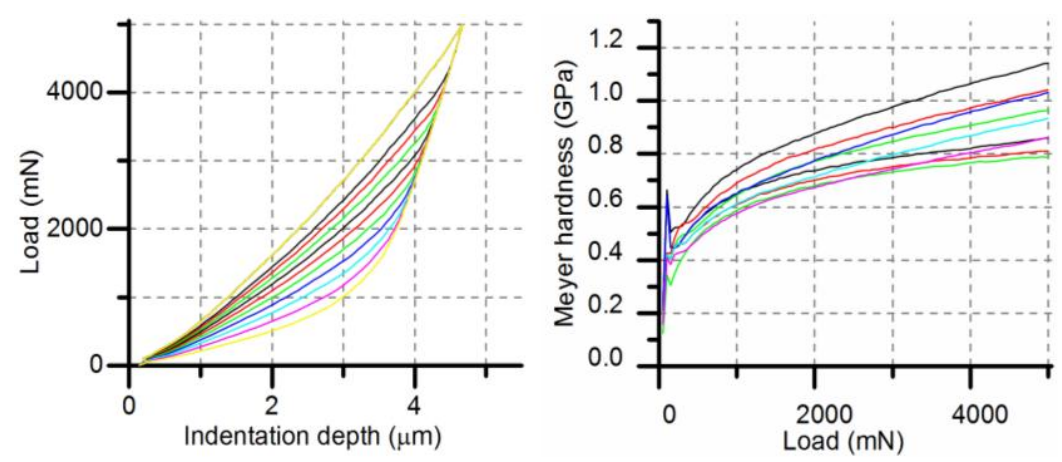

Fig. 2: Constructed axisymmetric finite element model of material and indenter. Full view (left) is shown also enlarged (right). 
In addition, for each material constants set, the geometry of indenter varied by changing the indenter's radius to $150,175,200 \mu \mathrm{m}$. This allows to find out how radius of indenter affects indentation results for different material's properties sets.

\section{Results and Discussions}

The output of finite element modelling includes nodal forces and displacements of all nodes in model, also contact forces and some other data. Using data of displacement of nodes in indenter's apex it is possible to plot a P-h diagram for this data set. All data sets of material properties used in calculations can be divided to three categories by the property that was modified from one set to another. These material properties include forward transformation stress $\left(\sigma_{\mathrm{F}}\right)$, reverse transformation stress $\left(\sigma_{\mathrm{R}}\right)$, maximum transformation

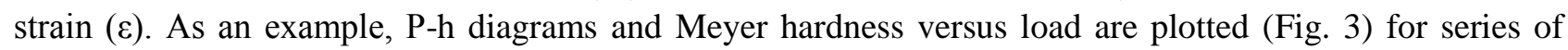
simulations with changing reverse transformation stress value while other properties were fixed.
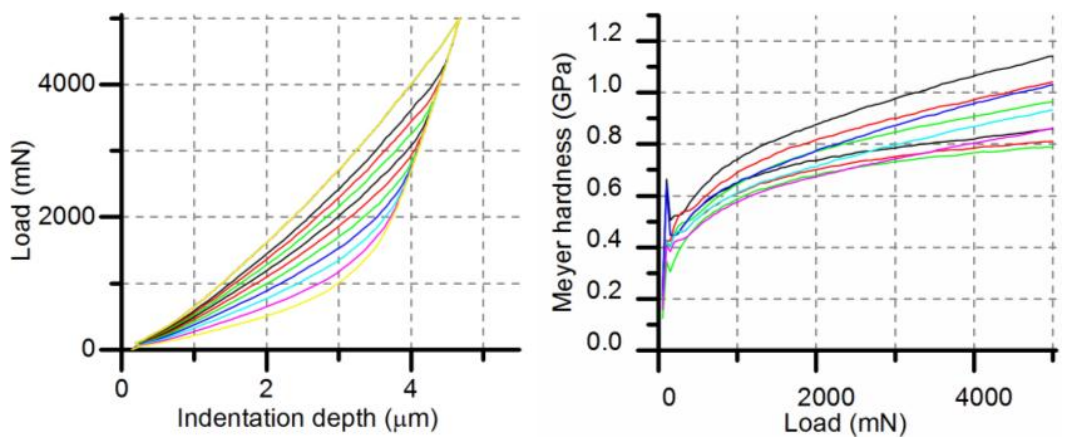

Fig. 3: P-h diagrams(left) and Meyer hardness(right). Each line represents material with different value of reverse transformation stress from 300 to $75 \mathrm{MPa}$.

For ordinary materials without SE properties, the most used characteristics of P-h diagram include constant of approximation in Kick's law, slope of unloading line in beginning of unloading and hardness. After analysis of obtained diagrams for superelastic materials the maximum indentation depth and ratio of unrecovered work to full work are found to be useful in characterization of acquired P-h diagrams. For first data set group (Fig. 4 a, b) the forward transformation stress was varied from 250 to 475 MPa by step of 25 $\mathrm{MPa}$. Maximum indentation depth gets significantly lower with increasing of transformation stress while changes in ratio of energies are moderate. Reverse transformation stresses are set to $200 \mathrm{MPa}$, maximum transformation strain is 0.05 for both directions of phase transformation. Young modulus is taken $45 \mathrm{GPa}$ for martensite phase and $75 \mathrm{GPa}$ for austenite and remain unchanged for all data sets presented in this paper. In second group (fig $4 \mathrm{c}, \mathrm{d}$ ) varied the reverse transformation stress from 300 to $75 \mathrm{MPa}$ by step of $25 \mathrm{MPa}$. Forward transformation stress is fixed at $250 \mathrm{MPa}$ and transformation strain is similar to previous group is 0.05. Relation of modified variable to ration of energies is almost linear with small dependence of indenter's radius. As expected, there are no changes in maximum indentation depth because during testing until maximum load is achieved, almost no material experiences an unloading. For last group (Fig. 4 e, f) forward and reverse transformation stresses are 350 and $200 \mathrm{MPa}$ respectively while transformation strain is changing from 0.01 to 0.10 totaling 10 points. Plot of energy ratios is similar with first group while plot of maximum indentation depth shows vivid correlation with indenter's radius.

\section{Connecting Function}

As can be seen from the plots (Fig. 4) and confirmed in calculation, plots of energy ration versus maximum transformation strain and forward transformation stress are close to hyperbolic, the remaining graph of energy ratios is linear. Having this in mind, we can propose the following function:

$$
\frac{E_{n}}{E_{t}}=-1.7 * 10^{-2}\left(\varepsilon_{t}+\frac{10 \sigma_{f}}{E}\right)^{-1}+73.3 \frac{\sigma_{r}}{E}
$$

On the picture (Fig. 5) shown the plot of approximating function against the input to demonstrate how much is the error between results of simulated indentation and a proposed function. Comparison to 
experimental results still has to be done, and it will help to find out how big is the error in estimating for real experiments.
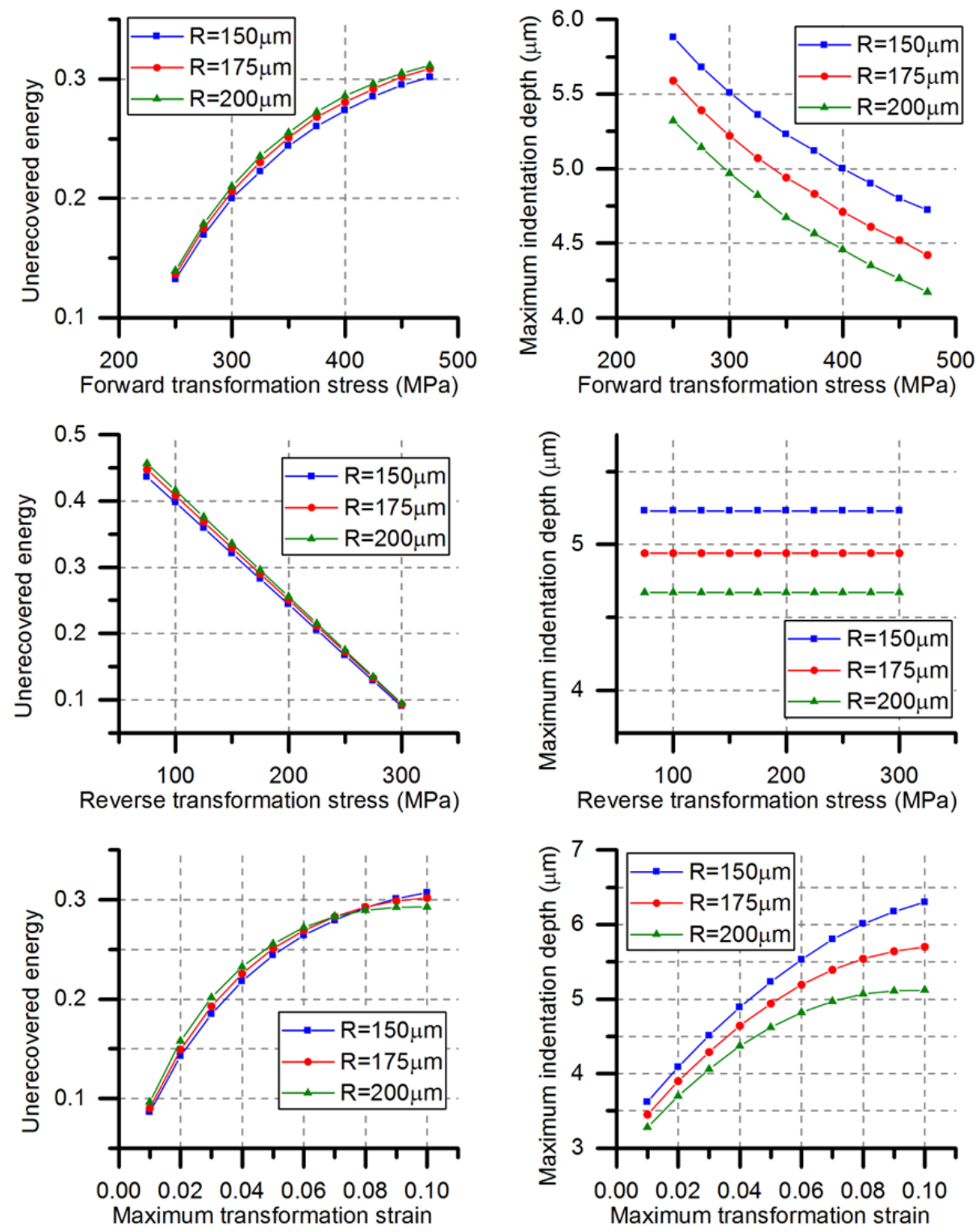

Figure 4. Calculated from simulated P-h diagrams values of recovered to total energy ratio and a maximum indentation depth. Each graph includes 3 lines for simulations with 150, 175, $200 \mu \mathrm{m}$ indenter radius.

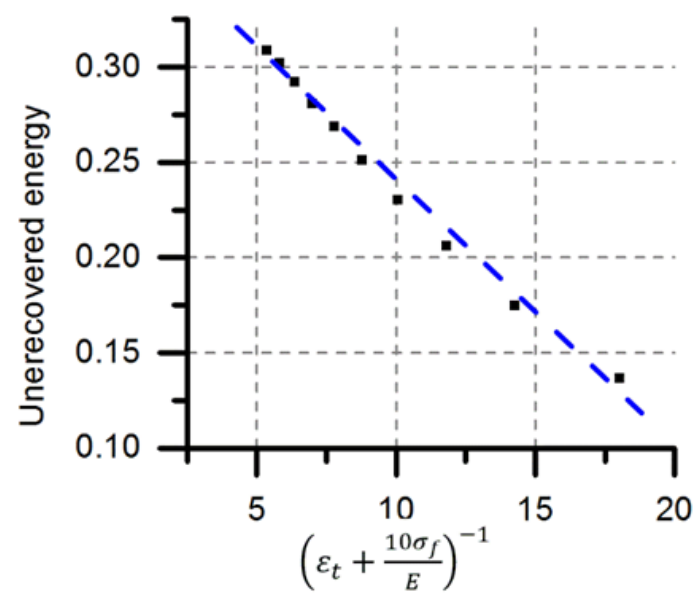

Fig. 5: Plot of energy ratio versus proposed member of equation. Dots are the simulated experimental data. 
Using function from this paper and relations for Young moduli and Stresses of transformation obtained by other authors in works [6] [7], it is possible to obtain all the properties shown on Fig. 1. This method have been tested on $55.9 \mathrm{Ni} 44.1 \mathrm{Ti}$ alloy which is superelastic at room temperature and the difference between mechanical properties obtained with uniaxial compression test and proposed technique is found to be below $20 \%$ for most specimens. It should be noted that these properties correspond to uniaxial compression rather than extension because indentation is mostly a compression process and does not accounts for material properties during extension. For example, as noted by several researches, a NiTi alloy may show a significant difference in behavior during uniaxial testing for extension and compression.

\section{Conclusion}

Constructed two-dimensional axisymmetric finite element model for spherical microindentation of SE materials. Based on this model several series of simulations performed with different material data set and different radius of indenter. With processed data from FEM, a connection function for determination of materials properties is proposed.

\section{References}

[1] Ölander, Arne. "An electrochemical investigation of solid cadmium-gold alloys." Journal of the American Chemical Society 54.10 (1932): 3819-3833, doi:10.1021/ja01349a004

[2] Wu, Ming H., and L. M. Schetky. "Industrial applications for shape memory alloys." Proceedings of the international conference on shape memory and superelastic technologies. 2000.

[3] Ribeiro, Ana Lúcia Roselino, et al. "Are new TiNbZr alloys potential substitutes of the Ti6Al4V alloy for dental applications? An electrochemical corrosion study." Biomedical Materials Vol. 8, No. 6, 2013, doi:10.1088/17486041/8/6/065005

[4] EVans, Aw G., and E. Arn Charles. "Fracture toughness determinations by indentation." Journal of the American Ceramic society Vol. 59 No.7, 1976, 371-372, doi: 10.1111/j.1151-2916.1976.tb10991

[5] Auricchio, Ferdinando. "A robust integration-algorithm for a finite-strain shape-memory-alloy superelastic model." International Journal of plasticity Vol. 17, No.7, 2001, 971-990, doi: 10.1016/S0749-6419(00)00050-4

[6] Yan, W., Sun, Q., Feng, X. Q., \& Qian, L. "Determination of transformation stresses of shape memory alloy thin films: a method based on spherical indentation." Applied physics letters Vol. 88, No.24, 2006, 241912, doi: $10.1063 / 1.2213018$

[7] Kan, Q., Yan, W., Kang, G., \& Sun, Q. "Oliver-Pharr indentation method in determining elastic moduli of shape memory alloys - A phase transformable material." Journal of the Mechanics and Physics of Solids Vol. 61, No.10, 2013, 2015-2033, doi: 10.1016/j.jmps.2013.05.007 